\title{
Re-irradiation plus hyperthermia for recurrent pediatric sarcoma; a simulation study to investigate feasibility
}

\author{
H. PETRA KOK ${ }^{1}$, IRMA W.E.M. VAN DIJK ${ }^{1}$, KOEN F. CRAMA ${ }^{1}$, \\ NICOLAAS A.P. FRANKEN ${ }^{1,2}$, COEN R.N. RASCH ${ }^{1}$, JOHANNES H.M. MERKS ${ }^{3,4}$, \\ JOHANNES CREZEE $^{1}$, BRIAN V. BALGOBIND ${ }^{1}$ and ARJAN BEL ${ }^{1}$
}

\author{
${ }^{1}$ Department of Radiation Oncology, Academic Medical Center, University of Amsterdam; ${ }^{2}$ Laboratory for Experimental \\ Oncology and Radiobiology (LEXOR)/Center for Experimental and Molecular Medicine; ${ }^{3}$ Department of Pediatric Oncology, \\ Emma Children's Hospital/Academic Medical Center, 1105 AZ Amsterdam; \\ ${ }^{4}$ Princess Máxima Center for Pediatric Oncology, 3584 CS Utrecht, The Netherlands
}

Received May 29, 2018; Accepted September 21, 2018

DOI: $10.3892 /$ ijo.2018.4622

\begin{abstract}
Recurrent pediatric tumors pose a challenge since treatment options may be limited, particularly after previous irradiation. Positive results have been reported for chemotherapy and hyperthermia, but the combination of re-irradiation and hyperthermia has not been investigated thus far, although it is a proven treatment strategy in adults. The theoretical feasibility of re-irradiation plus hyperthermia was investigated for infield recurrent pediatric sarcoma in the pelvic region and the extremities. A total of 46 recurrent pediatric sarcoma cases diagnosed at the Academic Medical Center (Amsterdam, The Netherlands) between 2002 and 2017 were evaluated. Patients not previously irradiated, outfield recurrences and locations other than the pelvis and extremities were excluded, ultimately yielding four eligible patients: Two with sarcomas in the pelvis and two in an extremity. Re-irradiation and hyperthermia treatment plans were simulated for $23 \times 2$ Gy treatment schedules and weekly hyperthermia. The radiosensitizing effect of hyperthermia was quantified using biological modelling with a temperature-dependent change in the parameters of the linear-quadratic model. The possible effectiveness of re-irradiation plus hyperthermia was estimated by calculating the equivalent radiotherapy dose distribution. Treatment planning revealed that tumors located in the pelvis and the extremities can be effectively heated in children. Equivalent dose distributions indicated
\end{abstract}

Correspondence to: Dr H. Petra Kok, Department of Radiation Oncology, Academic Medical Center, University of Amsterdam, Meibergdreef 9, 1105 AZ Amsterdam, The Netherlands

E-mail: h.p.kok@amc.uva.nl

Abbreviations: PTV, planning target volume; GTV, gross tumor volume; OAR, organ at risk; VMAT, volumetric modulated arc therapy

Key words: hyperthermia, re-irradiation, pediatrics, sarcoma, biological modelling that hyperthermic radiosensitization can be quantified as a target-selective additional D95\% of typically $10 \mathrm{~Gy}$, thereby delivering a possibly curative dose of $54 \mathrm{~Gy}$, without substantially increasing the equivalent dose to the organs at risk. Therefore, re-irradiation plus hyperthermia is a theoretically feasible and possibly effective treatment option for recurrent pediatric sarcoma in the pelvic region and the extremities, and its clinical feasibility is worthy of evaluation.

\section{Introduction}

Pediatric sarcomas represent $\sim 10 \%$ of all childhood malignancies (1). Treatment generally consists of a combination of surgery, chemotherapy and/or radiotherapy. Due to the continuously improving diagnostics and treatment techniques, the 5-year survival rate is currently $60-70 \%(2,3)$. However, the survival rate of pediatric sarcoma patients with recurrent disease is $<40 \%$ (4-6). The treatment options for this patient group are limited, since therapies are associated with (late) adverse events $(7,8)$, and applying a second curative treatment scheme may increase the risk of adverse events to unacceptable levels. This is particularly relevant for patients with local recurrence following previous radiotherapy.

Hyperthermia, i.e., heating of tumors to $39-43^{\circ} \mathrm{C}$ for $1 \mathrm{~h}$, is a treatment modality that may be combined with radiotherapy and/or chemotherapy to significantly enhance their effectiveness $(9,10)$. Several randomized trials in adults have demonstrated the effectiveness of hyperthermia in the control of soft tissue sarcomas and recurrent breast, cervix and head-neck carcinomas, among others, with no significant hyperthermia-related increase in side effects (11-17). For example, low-dose re-irradiation combined with hyperthermia applied for recurrent breast cancer can increase the complete response rate from 38 to $78 \%$, compared with re-irradiation alone (13).

Although hyperthermia is a clinically proven treatment for several tumors in adults (11-15), it is not commonly applied for pediatric tumors. The effectiveness of chemotherapy and hyperthermia has been investigated for children with sarcomas and germ cell tumors that respond poorly to or 
recur after chemotherapy (18). A phase II trial demonstrated that chemotherapy plus hyperthermia are successful as local therapy, with a 5-year survival of $52 \%$ (19). A prospective study for recurrent or refractory malignant non-testicular germ cell tumors further demonstrated the effectiveness of locoregional hyperthermia and chemotherapy, with a 5-year survival of $72 \%$ (20); the long-term prognosis for patients with poor response to therapy or after the first relapse was almost similar to that for patients receiving first-line treatment.

A possible role of radiotherapy plus hyperthermia for pediatric tumors has not been investigated thus far, despite the positive results of chemotherapy plus hyperthermia in pediatric patients and all successful clinical applications of hyperthermia combined with chemotherapy or radiotherapy in adults. This treatment combination may prove to be very effective for previously irradiated recurrent sarcomas, since a second curative dose would increase the risk of toxicity to unacceptable levels and the clinical feasibility of re-irradiation plus hyperthermia for adults with radiation-associated sarcoma has been demonstrated (21). Given the poor prognosis of pediatric patients with recurrent sarcoma, this treatment option is worth investigating further.

Before initiating a clinical feasibility study, the theoretical feasibility should be explored. Treatment planning combined with biological modelling is helpful for evaluating this theoretical feasibility. The radiosensitizing effect of hyperthermia may be considered as a local increase in tumor dose, which may be quantified using biological modelling, with a temperature-dependent change in the radiosensitivity parameters of the linear-quadratic model $(22,23)$. According to this concept, the possible effectiveness of re-irradiation and hyperthermia can be estimated by calculating the equivalent 3D dose distribution (24-26), i.e., the radiation dose that exerts a biological effect equivalent to that of the combined re-irradiation plus hyperthermia treatment.

The aim of the present study was to investigate the theoretical feasibility of re-irradiation plus hyperthermia for infield recurrent pediatric sarcomas. Re-irradiation and hyperthermia treatment plans were simulated and equivalent 3D dose distributions were calculated and evaluated based on dose-volume histograms (DVH).

\section{Materials and methods}

Patient selection. In the Emma Children's Hospital - Academic Medical Center (Amsterdam, The Netherlands), 46 pediatric patients (aged $<18$ years) were diagnosed with recurrent sarcoma (29 Ewing's sarcomas, 10 rhabdomyosarcomas and 7 non-rhabdomyosarcoma soft tissue sarcomas) between 2002 and 2017, 41 of which developed after previous irradiation and 11 were infield recurrences. Only pelvic and extremity tumors were included, due to our ample experience with clinical hyperthermia for these locations in adults. Bulky pelvic target volumes with a diameter $>15 \mathrm{~cm}$ were excluded, since locoregional hyperthermia at $70 \mathrm{MHz}$ (see subsection 'Hyperthermia treatment planning') yields a heating focus of $\sim 10-15 \mathrm{~cm}$ and, thus, effective heating of bulkier volumes is difficult. This selection process resulted in 2 patients with a recurrent pelvic tumor and 2 patients with a recurrence in an extremity (Fig. 1).
Recurrent pelvic sarcomas

Patient 1. A 3-year-old male patient presented with recurrent embryonal rhabdomyosarcoma, located perivesically in the left side of the pelvis. The tumor was first diagnosed at the age of 1 year. The primary tumor was treated with chemotherapy, followed by resection and proton radiotherapy (50.4 Gy in 28 fractions) combined with pulmonary radiotherapy due to lung metastases.

Patient 2. A 17-year-old female patient presented with a second recurrence of a pararectal alveolar rhabdomyosarcoma. The primary tumor was diagnosed at the age of 15 years and treated with radiotherapy (54 Gy; 30x1.8 Gy) and chemotherapy. The first recurrence at 16 years of age was located in both breasts and the left inguinal area, and was treated with radiotherapy [41.4 Gy; 23x1.8 Gy, followed by a boost to $50.4 \mathrm{~Gy}$ for positron emission tomography (PET)-positive breast lesions].

\section{Recurrent extremity sarcomas}

Patient 3. A 14-year-old male patient presented with recurrent Ewing's sarcoma in the second metatarsal bone of the right foot. The first diagnosis was at 12 years of age and treatment consisted of chemotherapy, followed by resection and postoperative radiotherapy (54 Gy; 30x1.8 Gy). Lung metastases were resected, followed by pulmonary radiotherapy (15 Gy; $10 x 1.5 \mathrm{~Gy})$.

Patient 4. A 13-year-old male patient presented with recurrent alveolar rhabdomyosarcoma in the left lower leg. The tumor was first diagnosed at 11 years of age at an advanced stage, with lymph node (inguinal, iliac and para-aortal), bone and lung metastases. Initial treatment consisted of chemotherapy followed by radiotherapy to all primary locations. The location of the recurrence initially received $54 \mathrm{~Gy}(30 \mathrm{x} 1.8 \mathrm{~Gy})$.

\section{Treatment simulations}

Radiotherapy treatment planning. External beam re-irradiation treatment plans for an Elekta Agillity 10MV machine were created using the diagnostic computed tomography scans. The gross tumor volume (GTV), planning target volume (PTV) and organs at risk (OARs; femur, rectum and bladder), if relevant, were delineated. Treatment plans were created for a re-irradiation schedule of $23 \times 2$ Gy and weekly hyperthermia, as applied clinically for recurrent breast cancer. Treatment planning was performed at $2 \times 2 \times 2 \mathrm{~mm}^{3}$ resolution using Raystation (version 6.0; RaySearch Labs, Stockholm, Sweden). Volumetric modulated arc therapy (VMAT) plan optimizations were started with objective values that were individually optimized to minimize OAR dose, while maintaining ICRU-based PTV coverage (D98\% >95\%, D2\% <107\%) (27).

Hyperthermia treatment planning. Hyperthermia treatment of pelvic tumors was simulated for the $70 \mathrm{MHz}$ AMC-4 system, which consists of a ring of four waveguides (fixed aperture size $20 \times 34 \mathrm{~cm}^{2}$ ) positioned around the patient (28). For the extremities, the $70 \mathrm{MHz}$ AMC-2 system was simulated, consisting of two waveguides positioned at the top and bottom of the target (29). Waveguides with different aperture sizes may be selected for this system, and an aperture size of $20 \times 34 \mathrm{~cm}^{2}$ was selected. For both systems, 


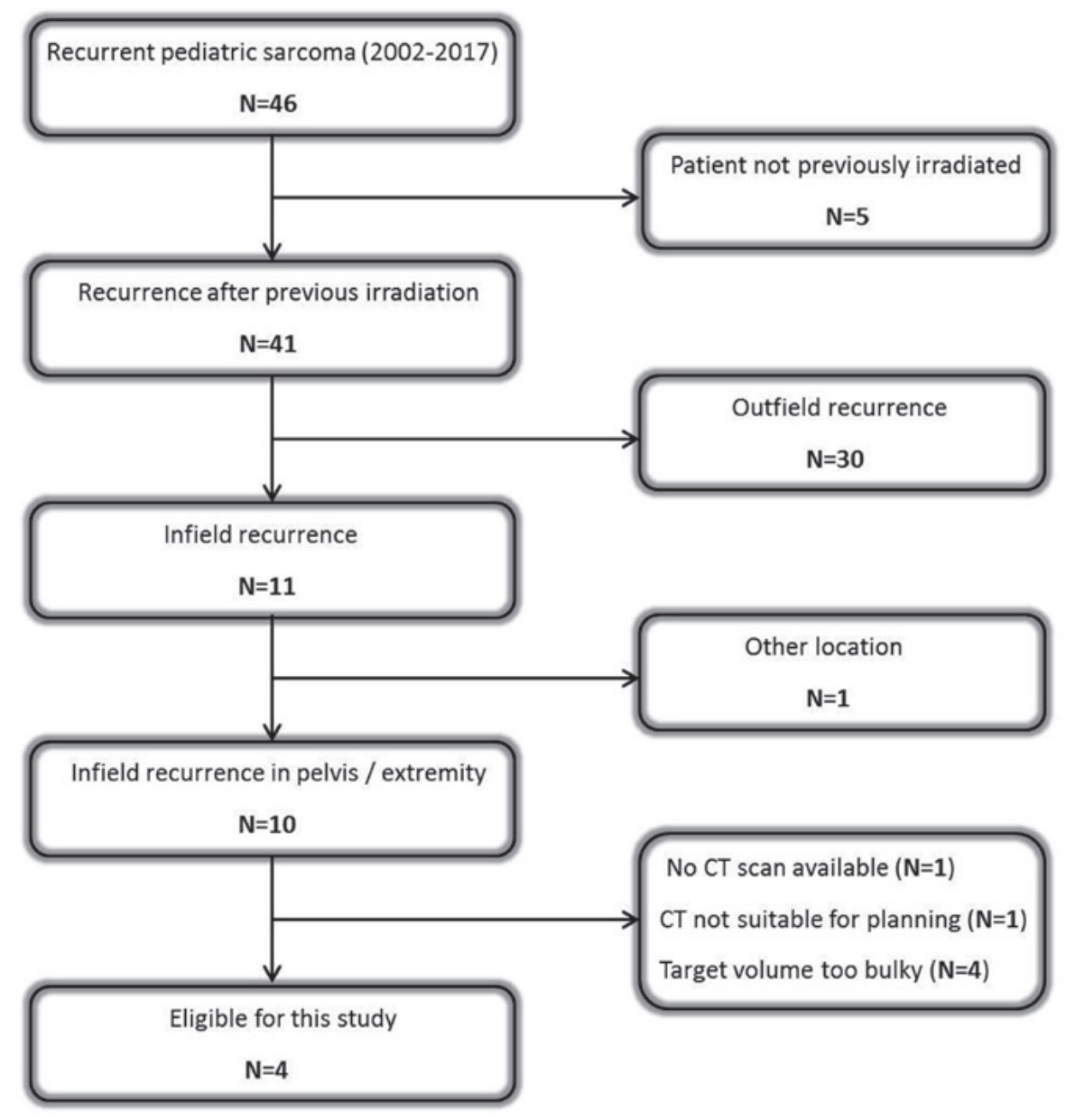

Figure 1. Flow chart of the patient selection process. CT, computed tomography.

water boluses provide skin cooling and coupling of the electromagnetic energy into the tissue. Following regular clinical practice, aggressive cooling (bolus temperature $12^{\circ} \mathrm{C}$ ) was simulated for deep heating of the pelvis to avoid overheating of the skin, and a higher bolus temperature $\left(42^{\circ} \mathrm{C}\right)$ was modelled for heating the extremities, since the skin is part of the target volume. The set-up for pelvic and extremity tumors is shown in Fig. 2. Hyperthermia treatment planning was performed using Plan2Heat (30). Hounsfield unit-based tissue segmentation into muscle, fat, bone and air was combined with delineations to create a patient model, which was inserted in the applicator model for the AMC-4 or AMC-2 system. Literature-based dielectric and thermal properties were assigned, and electric field and thermal computations were performed using finite difference methods $(30,31)$ at $2.5 \times 2.5 \times 2.5 \mathrm{~mm}^{3}$ (pelvis) or $1 \times 1 \times 2.5 \mathrm{~mm}^{3}$ (extremities). Temperature-based optimization was performed to maximize the $T_{90}$, i.e., the temperature achieved in at least $90 \%$ of the PTV, with hard constraints of $45^{\circ} \mathrm{C}$ to all normal tissues $(30,32)$, since a pain sensation is experienced when tissue temperatures exceed $45^{\circ} \mathrm{C}(33)$.

Biological modelling. Biological evaluation of the combined radiotherapy and hyperthermia treatment was performed using the in-house developed X-Term software package, which calculates the $3 \mathrm{D}$ equivalent radiotherapy dose distribution $\left(\mathrm{EQD}_{\mathrm{RT}}\right)$ forauser-specifiedtreatmentschedule(26). Calculations are based on the linear-quadratic (LQ) model, expressing the survival fraction (SF) of cells after delivering $n$ fractions with a fraction dose $d$ (Gy) according to the following equation:

$$
S F(n, d, \alpha, \beta)=e^{-n\left(\alpha d+\beta d^{2}\right)},
$$

where $\alpha\left(\mathrm{Gy}^{-1}\right)$ and $\beta\left(\mathrm{Gy}^{-2}\right)$ are the radiosensitivity parameters. X-Term uses an extension of the LQ-model, in which $\alpha$ and $\beta$ depend on the temperature and the time interval between radiotherapy and hyperthermia. Additionally, X-Term accounts for the direct hyperthermic cytotoxicity in tumor tissue (34). Mathematical functions were parametrized such that the $\mathrm{EQD}_{\mathrm{RT}}$ depends mainly on the $\alpha / \beta$ ratio and on the hyperthermia-enhancing factors, rather than on the individual parameters (34). More details on the software package, mathematics and the derivation of the temperature dependency of $\alpha$ and $\beta$ may be found in earlier publications $(26,34)$.

Weekly application of hyperthermia directly after the first radiotherapy fraction was modelled. Ratios $\alpha / \beta=10$ Gy and $\alpha / \beta=3$ Gy were assumed for tumor and OARs, respectively, under non-hyperthermic conditions $\left(37^{\circ} \mathrm{C}\right)$. Since curative radiation schedules for primary sarcomas typically use a fraction dose of $1.8 \mathrm{~Gy}$, the reference fraction dose for all $\mathrm{EQD}_{\mathrm{RT}}$ calculations was $1.8 \mathrm{~Gy}$. 


\section{Heating pelvic tumors}

AMC-4 SYSTEM

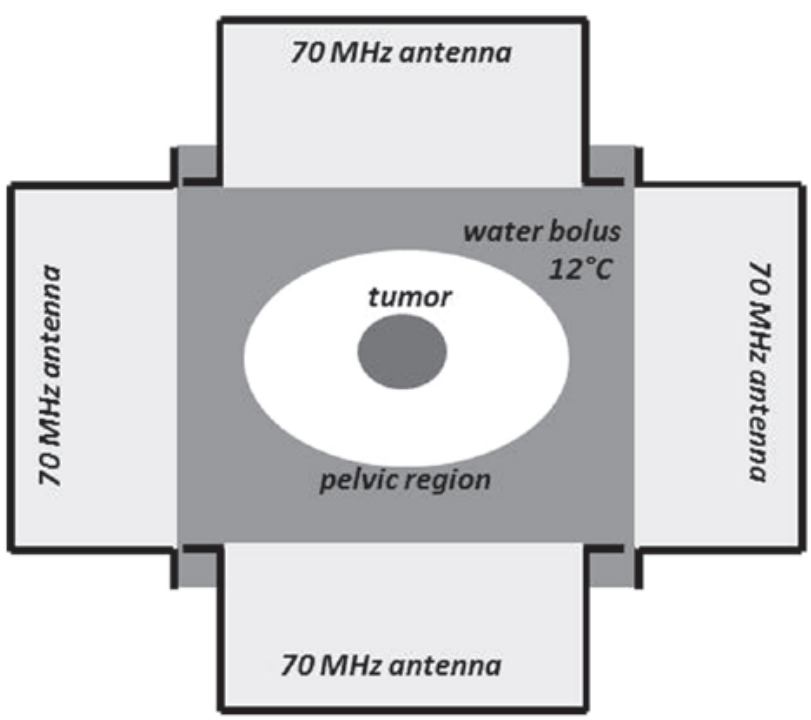

Heating extremity tumors

AMC-2 SYSTEM

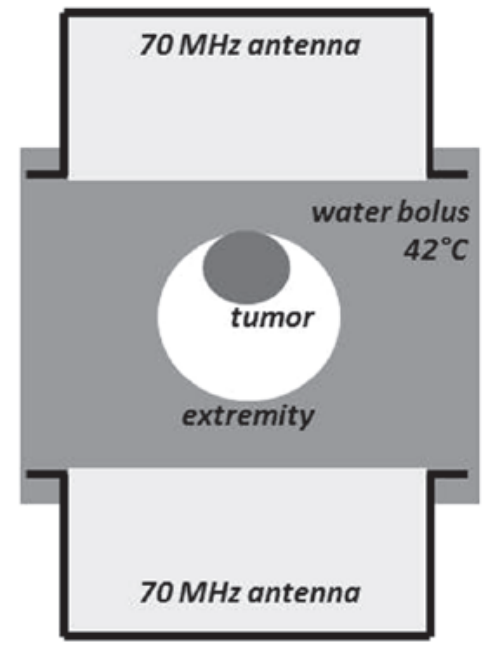

Figure 2. Schematic heating of pelvic and extremity tumors.

The reliability of biological modelling is affected by the statistical accuracy of the model parameters. Therefore, a $95 \%$ confidence interval for the parameters was translated into a confidence interval for $\mathrm{EQD}_{\mathrm{RT}}$, as described by van Leeuwen et al (34).

Statistical analysis. The planned temperature distributions were evaluated by indexed temperatures $\mathrm{T}_{10}, \mathrm{~T}_{50}$ and $\mathrm{T}_{90}$, i.e., the temperature achieved in at least 10, 50 and $90 \%$ of the PTV, following the quality assurance guidelines (35). Ideally, the $\mathrm{EQD}_{\mathrm{RT}}$ should approach the standard curative dose (54 Gy). The EQD ${ }_{\mathrm{RT}}$ distributions were analyzed using standard DVH parameters. The increase in D98\% and D95\% by adding hyperthermia was evaluated for the PTV (36). Additionally, the increase in D2\% for the OARs was evaluated (36).

\section{Results}

Recurrent pelvic sarcomas. For patient 1 (3-year-old male) the planned PTV temperatures $\mathrm{T}_{10}, \mathrm{~T}_{50}$ and $\mathrm{T}_{90}$ were 43.9, 42.7 and $41.1^{\circ} \mathrm{C}$, respectively, with therapeutic temperatures between 39 and $43^{\circ} \mathrm{C}$. The total absorbed power in the patient was 168 W. Fig. 3 shows the radiation dose distribution, converted to a reference fraction dose of $1.8 \mathrm{~Gy}$, the temperature distribution, the predicted $\mathrm{EQD}_{\mathrm{RT}}$ of the combined treatment and the DVH for the original and equivalent radiation dose distributions. A substantial increase in equivalent dose for the PTV is predicted, with a considerably lower increase in equivalent OAR dose. The D98\% in the PTV is predicted to increase from 44.4 to $51.6 \mathrm{~Gy}$; the $\mathrm{D} 95 \%$ increases from 45.4 to $53.4 \mathrm{~Gy}$, which approaches the curative dose of $54 \mathrm{~Gy}$. Although the rectum and bladder are located within the PTV, the effect is rather tumor-selective: the D2\% increases from 49.2 to 54.3 Gy and from 49.0 to 53.8 Gy for the bladder and rectum, respectively; i.e., approximately half of the increase in $\mathrm{D} 95 \%$.

For patient 2 (17-year-old female) the planned PTV temperatures $\mathrm{T}_{10}, \mathrm{~T}_{50}$ and $\mathrm{T}_{90}$ were $41.8,40.8$ and $39.5^{\circ} \mathrm{C}$, respectively. The total absorbed power in the patient was $471 \mathrm{~W}$. Fig. 4 shows the dose and temperature distributions. Although therapeutic heating is possible, achievable temperatures are lower compared with those for patient 1 , which is also reflected by a lower enhancement in $\mathrm{EQD}_{\mathrm{RT}}$. The $\mathrm{D} 98 \%$ increases from 41.7 to 45.3 Gy and the D95\% increases from 44.2 to $48.3 \mathrm{~Gy}$. The overlap of the OAR (bladder) with the PTV is very small; thus, the predicted $\mathrm{EQD}_{\mathrm{RT}}$ in the bladder is practically the same as the original dose.

Recurrent extremity sarcomas. For patient 3 (foot; 14-year-old male) the planned PTV temperatures $\mathrm{T}_{10}, \mathrm{~T}_{50}$ and $\mathrm{T}_{90}$ were $43.3,42.2$ and $41.4^{\circ} \mathrm{C}$, respectively. The total absorbed power in the patient was 19 W. Fig. 5 shows the radiation dose distribution, converted to a reference fraction dose of $1.8 \mathrm{~Gy}$, the temperature distribution, the $\mathrm{EQD}_{\mathrm{RT}}$ of the combined treatment and the DVHs for the original and equivalent radiation dose distributions. A substantial increase in equivalent PTV dose is predicted: the D98\% increases from 28.0 to 38.2 Gy and the D95\% increases from 35.0 to $46.1 \mathrm{~Gy}$. As a result of skin sparing in the extremities, the D98\% and D95\% of the original PTV dose are quite low, reflecting an underdosage of the skin. Adding hyperthermia yields a substantial increase of the equivalent dose in this region, while no substantial additional toxicity in the healthy skin is expected due to the tumor selectivity of hyperthermia. For the GTV, the D98\% and D95\% increase from 46.4 to $54.4 \mathrm{~Gy}$ and from 46.5 to $54.7 \mathrm{~Gy}$, respectively, thereby realizing a curative dose of $54 \mathrm{~Gy}$.

For patient 4 (lower leg; 13 -year-old male) the planned temperatures $\mathrm{T}_{10}, \mathrm{~T}_{50}$ and $\mathrm{T}_{90}$ were $44.2,43.0$ and $41.3^{\circ} \mathrm{C}$. The 

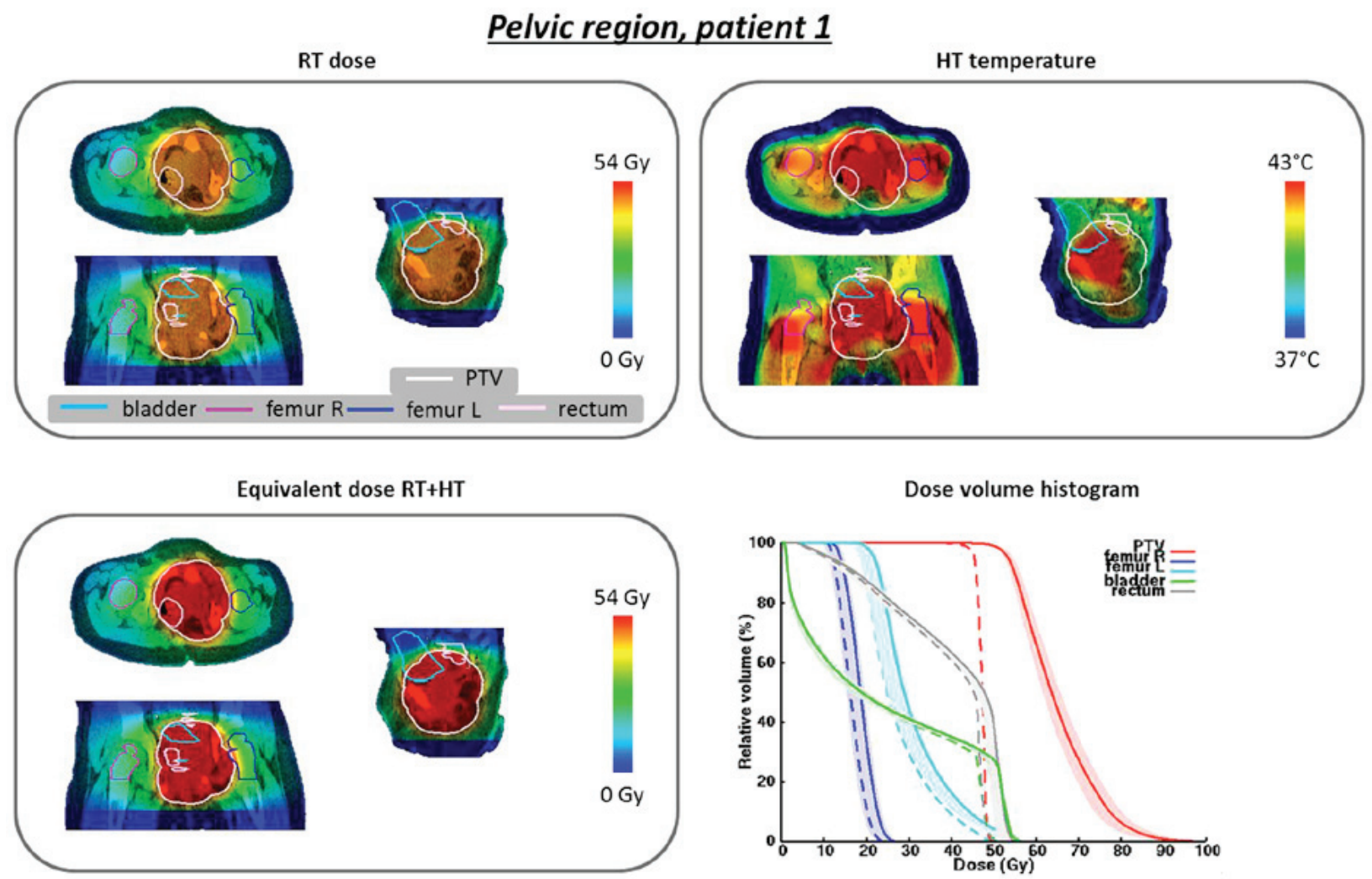

Figure 3. Radiation dose distribution (RT dose) for the 3-year-old male patient with recurrent pelvic sarcoma, showing the temperature distribution (HT temperature), the $\mathrm{EQD}_{\mathrm{RT}}$ for the combined treatment (equivalent dose $\mathrm{RT}+\mathrm{HT}$ ) and the dose-volume histograms for radiation alone (dotted lines) and the combined treatment (solid lines). The reference fraction dose is $1.8 \mathrm{~Gy}$. RT, radiotherapy; HT, hyperthermia; PTV, planning target volume.

\section{Pelvic region, patient 2}

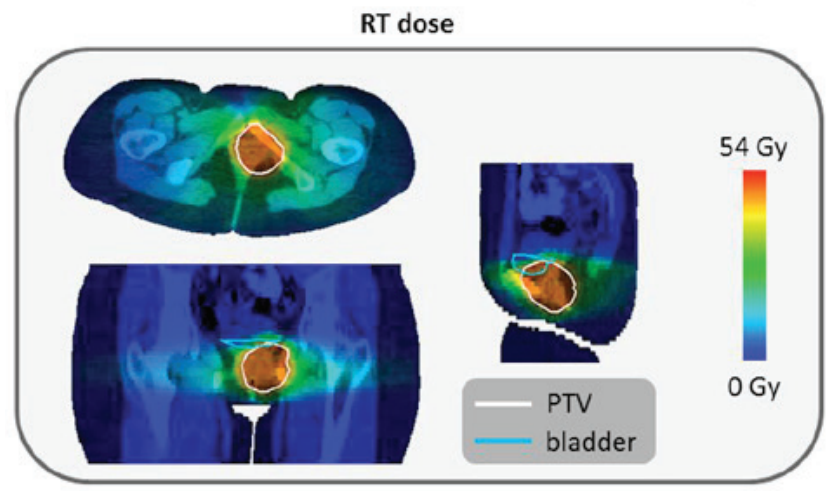

Equivalent dose RT+HT

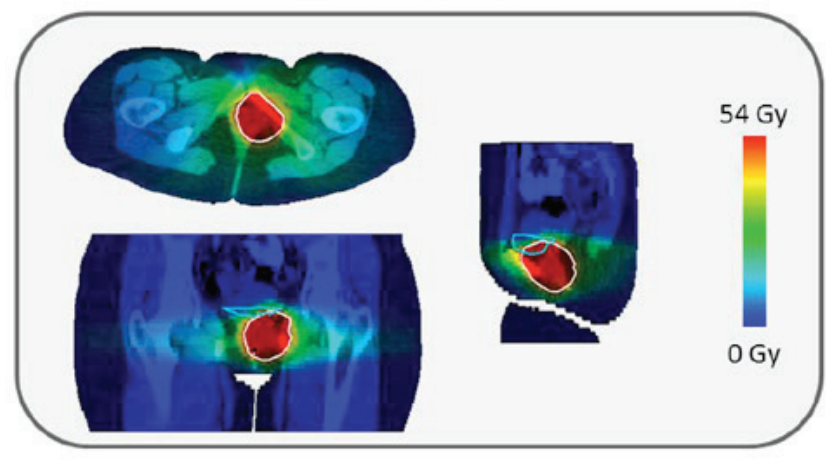

HT temperature

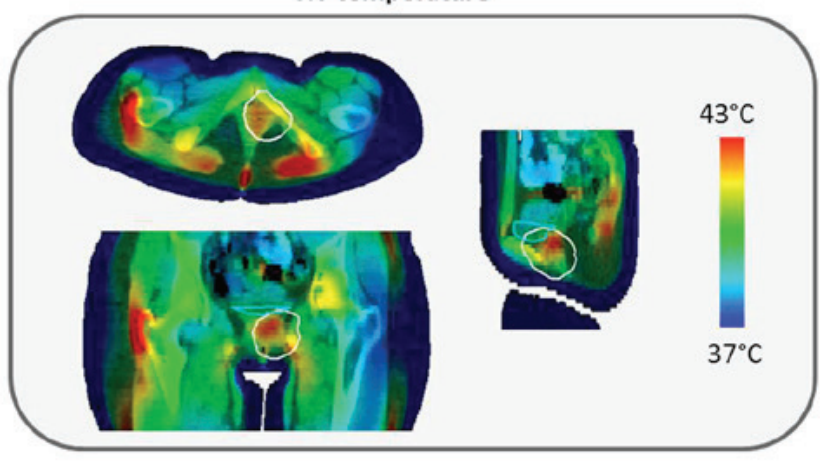

Dose volume histogram

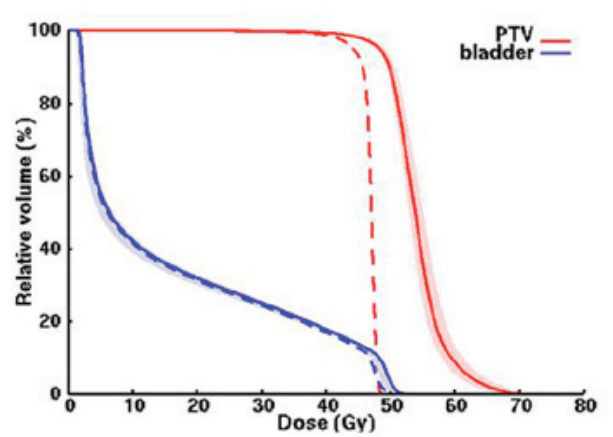

Figure 4. Radiation dose distribution (RT dose) for the 17-year-old female patient with recurrent pelvic sarcoma, showing the temperature distribution (HT temperature), the $\mathrm{EQD}_{\mathrm{RT}}$ for the combined treatment (equivalent dose $\mathrm{RT}+\mathrm{HT}$ ) and the dose-volume histograms for radiation alone (dotted lines) and the combined treatment (solid lines). The reference fraction dose is $1.8 \mathrm{~Gy}$. RT, radiotherapy; HT, hyperthermia; PTV, planning target volume. 


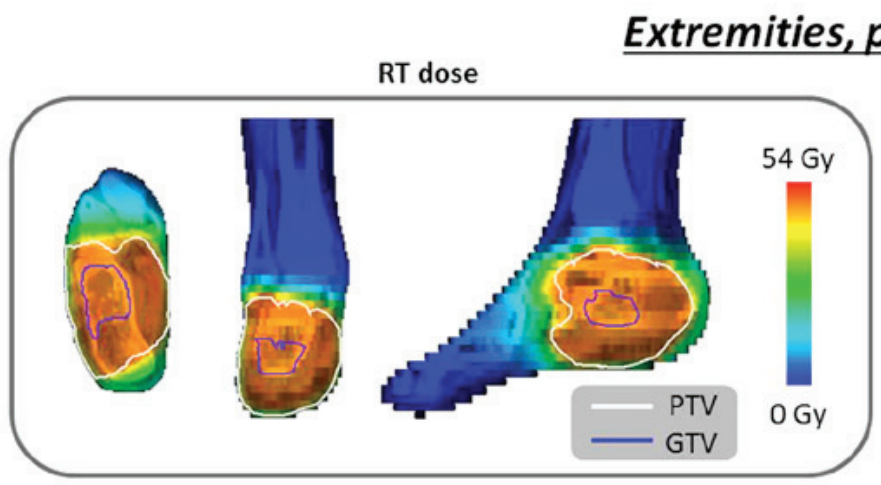

HT temperature
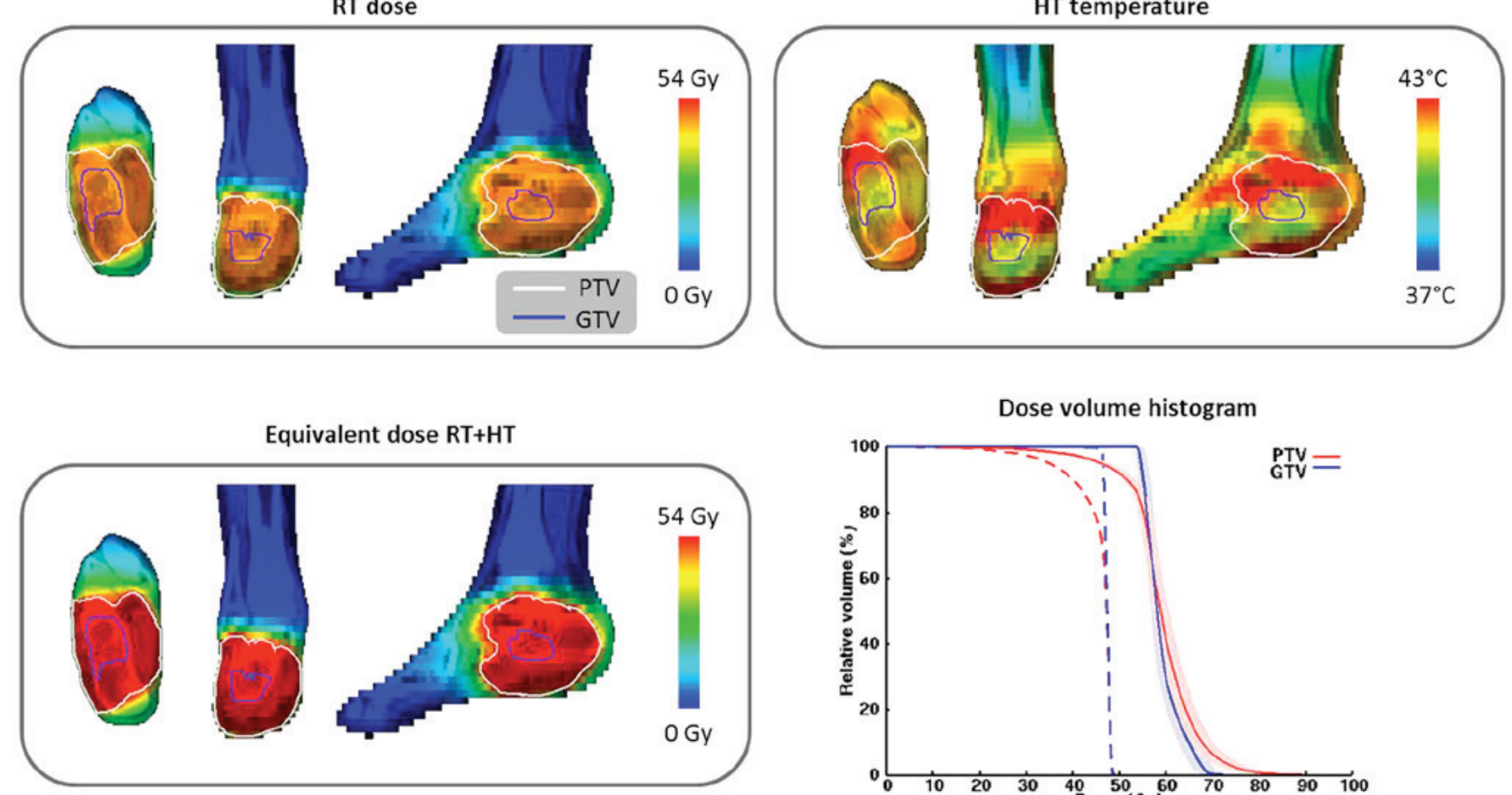

Dose volume histogram

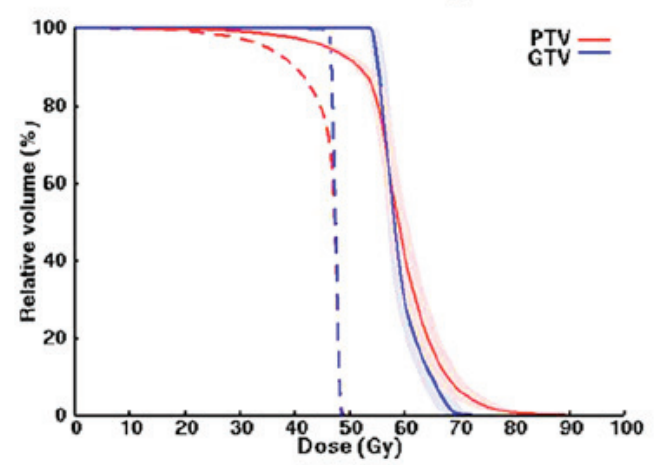

Figure 5. Radiation dose distribution (RT dose) for the 14-year-old male with recurrent sarcoma of the right foot, showing the temperature distribution (HT temperature), the $\mathrm{EQD}_{\mathrm{RT}}$ for the combined treatment (equivalent dose $\mathrm{RT}+\mathrm{HT}$ ) and the dose-volume histograms for radiation alone (dotted line) and the combined treatment (solid line). The reference fraction dose is $1.8 \mathrm{~Gy}$. RT, radiotherapy; HT, hyperthermia; PTV, planning target volume; GTV, gross tumor volume.

\section{Extremities, patient 4}
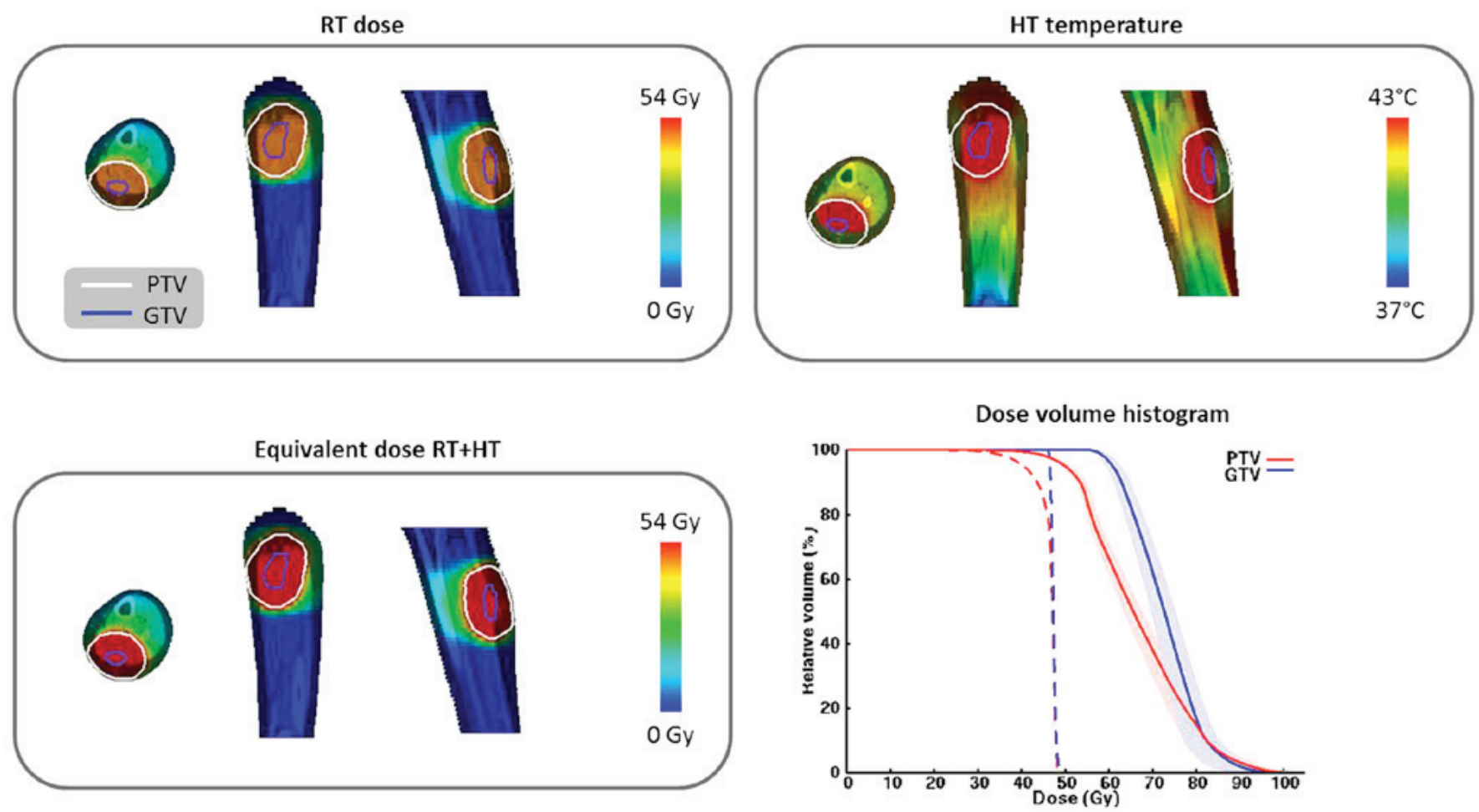

Figure 6. Three orthogonal slices of the radiation dose distribution (RT dose) for the 13-year-old male with recurrent sarcoma of the leg, showing the temperature distribution (HT temperature), the $\mathrm{EQD}_{\mathrm{RT}}$ for the combined treatment (equivalent dose $\mathrm{RT}+\mathrm{HT}$ ) and the dose-volume histograms for radiation alone (dotted line) and the combined treatment (solid line). The reference fraction dose is $1.8 \mathrm{~Gy}$. RT, radiotherapy; HT, hyperthermia; PTV, planning target volume; GTV, gross tumor volume. 
total absorbed power in the patient was $61 \mathrm{~W}$. Fig. 6 shows the dose and temperature distributions. The D98\% in the PTV increases from 36.5 to $45.8 \mathrm{~Gy}$ and the D95\% increases from 40.6 to 50.0 Gy. For the GTV, the D98\% and D95\% increase from 46.3 to 58.9 Gy and from 46.5 to 60.9 Gy, respectively, again realizing a curative dose.

\section{Discussion}

This simulation study demonstrates that re-irradiation plus hyperthermia may be a feasible treatment combination for infield recurrent pediatric sarcomas in the pelvic region or the extremities. 3D equivalent dose calculations combining clinically representative dose and temperature distributions predicted that radiosensitization by hyperthermia yields an increase in equivalent D95\% of typically $10 \mathrm{~Gy}$, thereby delivering a possible curative dose without a substantial additional risk of normal tissue toxicity. This treatment combination may thus improve the currently disappointing survival rate of sarcoma patients with infield recurrence and warrants further clinical evaluation. The long-term effectiveness and possible late complications of this treatment combination in pediatric recurrent sarcoma patients must be assessed in a clinical study.

An important aspect of biological modelling is the choice of the parameter values used. The value of $\alpha / \beta$ for tumor tissue may vary between different pathologies (37). The present study assumed the commonly applied value of $\alpha / \beta=10$ Gy for tumor tissue under normothermic conditions. The temperature-dependent behavior of the enhancement factor of $\alpha / \beta$ was estimated based on detailed experiments for cervical cancer cells, as described in an earlier study (34). The accuracy of the prediction of the equivalent dose would be expected to improve if more exact parameter values were available, but derivation of such parameter values is very challenging; thus, a commonly used value was adopted in this study. Although the exact increase in equivalent dose may be somewhat different when the precise parameters are known, the overall conclusion that hyperthermia can strongly increase the equivalent tumor dose is not likely to change substantially. A clinically relevant enhancement in radiosensitivity has also been demonstrated in clinical studies when adding hyperthermia for cervical cancer $(\alpha / \beta>10$ Gy) and (recurrent) breast cancer $(\alpha / \beta<10 \mathrm{~Gy})(12,13)$. Biological modelling studies evaluating radiotherapy plus hyperthermia for cervical and prostate cancer also predicted the same order of magnitude increase in equivalent tumor dose $(24,25)$. The radiosensitization effect by hyperthermia is thus more determinant of the increase in equivalent radiation dose than the exact initial reference value of $\alpha / \beta$. Realistic uncertainties in hyperthermic radiosensitization were included in the modelling, as reflected by the confidence intervals.

Regarding normal tissue and OARs, the conventional value of $\alpha / \beta=3$ Gy for late effects was applied, as commonly used for biological modelling in both adults and children $(38,39)$. Clinical studies combining radiotherapy and hyperthermia in adults for several tumor sites have demonstrated that the toxicity in normal tissue is generally not significantly increased compared with radiation or re-irradiation alone $(12,13)$. Although there is no reason to hypothesize that normal tissue toxicity will increase in children, it is currently unknown whether this value of $\alpha / \beta$ is fully appropriate for pediatric patients, as the OARs at a young age may behave radiobiologically differently compared with the same OARs in adults. Nevertheless, most relevant and determining for the effect of re-irradiation plus hyperthermia is the increase in equivalent radiation dose by adding hyperthermia, which is determined more by the radiosensitization due to hyperthermia rather than by the initial normothermic reference value of $\alpha / \beta$.

There are usually some challenges regarding the maximum cumulative dose in normal tissue when applying re-irradiation (40). Although the hyperthermic enhancement of the radiation dose is already predicted to be very low in normal tissue in the examples discussed in this study, a time interval (e.g., 1 h) between radiotherapy and hyperthermia may be applied to further minimize the enhancement in normal tissue, if desired $(41,42)$. This may be required when there is a risk of exceeding the tolerance dose to a specific OAR, in case of slowly proliferating tissues (e.g., bone and central nervous system) receiving a relatively high cumulative dose, or in case the time interval between the initial radiation treatment and re-irradiation is relatively short. Predictably, applying a time interval will also somewhat reduce the equivalent tumor dose, and biological modelling may be helpful in determining a good balance, such that a sufficiently high tumor dose can still be expected.

The results exhibited some variation regarding increased effectiveness among the four patients, which is due to differences in heatability. With locoregional hyperthermia, excessive temperatures (hot spots) in normal tissue may develop at tissue interfaces (e.g., fat, muscle and bone) due to the differences in tissue properties $(43,44)$. These hot spots should be avoided and, therefore, all tissue temperatures were constrained to $45^{\circ} \mathrm{C}$, which can limit the maximum achievable target temperature. Extremity tumors are easier to heat compared with pelvic tumors, due to their smaller anatomical size and the lower number of tissue interfaces at which hot spots may occur. Due to the differences in heatability between patients, it is advisable to perform pre-planning to determine whether effective heating is possible (i.e., $\left.\mathrm{T}_{90}>39^{\circ} \mathrm{C}\right)(45,46)$, prior to deciding clinical treatment with re-irradiation and hyperthermia. Particularly for relatively large tumor volumes, and in case of multiple treatment options, it should be determined first whether sufficient target coverage can be achieved.

When pre-planning indicates that the target region cannot be effectively heated and the treatment intent is still curative, re-irradiation plus whole-body hyperthermia may be considered. There is some, albeit limited, clinical experience with whole-body heating for children in combination with chemotherapy (47-49). Whole-body heating requires a dedicated heating device, which heats the patient to a maximum of $41.8^{\circ} \mathrm{C}$. This ensures a homogeneous target temperature and, thus, effective enhancement ( 10 Gy) of the radiotherapy. Whole-body hyperthermia is not the first choice of treatment, however, since deep analgesia and sedation or general anesthesia are required, and greater efforts are needed (including intensive medical care) compared with locoregional hyperthermia.

The AMC-4 system, as it is extensively used for adults $(12,50,51)$, was modelled for heating pelvic tumors. 
The 17-year-old patient had the body sizes of a small adult, and the 3-year-old patient had a tumor located relatively low in the pelvis; therefore, heating with the AMC-4 system was considered feasible. However, the axial length of the patient covered by the waveguides and boluses is $40 \mathrm{~cm}$, which may not be feasible for very small children or tumors located relatively high in the pelvis. In those cases, a system with smaller antennas would be desired, e.g., the BSD Sigma-30 (Pyrexar Medical, Salt Lake City, UT, USA) (20,52). However, this system operates at a higher frequency, yielding a smaller heating focus. Additionally, a higher frequency (typically 90-130 MHz) also improves the steering capabilities, which would possibly allow further reduction of normal tissue heating, for example of the femoral heads for patient 1 . Pre-planning should evaluate whether and to which extent this affects target coverage.

Sarcomas often contain hypoxic regions with reduced radiosensitivity. Hypoxic tumors are more sensitive to hyperthermia (53) and the mechanism responsible for part of the apparent hyperthermic radiosensitization of hypoxic tumors is direct cell kill. The rate of direct cell kill depends on the oxygenation status. A strong dose-effect relationship exists, both for direct cytotoxicity and for radiosensitization, due to inhibition of DNA repair by hyperthermia (54-56). Enhanced direct cytotoxicity was not accounted for in the present study, since no information on the oxygenation status was available. Therefore, the predicted increase in equivalent dose is likely an underestimation of the real enhancement. The accuracy of biological treatment planning may be improved by including hypoxia in the models (57). This is subject of further research and requires determination of the oxygenation status and tumor reoxygenation in individual patients by hypoxia PET/MRI techniques $(58,59)$.

Additionally, the tissue properties (fibrosis, necrosis) and tumor perfusion may also be altered in previously irradiated tissues, which may affect the temperature distribution and, thereby, the equivalent dose calculations. Incorporating these influences on pre-irradiated tissue in the X-Term calculation models requires further research as well as dedicated imaging techniques.

To avoid complications due to excessive temperatures developing in normal tissue, temperature feedback during treatment is crucial. For adults, standard (minimally) invasive thermometry probes are placed in or close to the tumor and normal tissue heating is monitored by patient feedback. When tissue temperatures exceed $45^{\circ} \mathrm{C}$, a pain sensation is experienced (33) and the operator changes the system settings to resolve the hot spot (60). In general, this procedure should also be feasible for children; however, anesthesia might be required for very young children and exact hot spot locations cannot be communicated. Sedation does not increase the risk of burns at elevated temperatures (20). For those cases, 3D non-invasive thermometry feedback by MR is desired, particularly in the pelvis. Additionally, some studies suggest that heating pelvic malignancies in pediatric patients may increase the risk of osteonecrosis or avascular necrosis (AVN) $(61,62)$. Young age is also considered a possible risk factor for AVN $(61,62)$. Therefore, high temperatures in the femoral heads should be avoided. This may be achieved by setting more strict temperature constraints to the femoral heads when optimizing antenna settings and/or using a higher operating frequency, as mentioned above.

MR-thermometry is not generally available and, based on the previous considerations, a first clinical feasibility study evaluating re-irradiation plus hyperthermia for infield recurrent pediatric sarcomas will be initiated for patients with pelvic malignancies not requiring anesthesia, and extremity sarcomas. For the latter category, age will be no limitation, since OARs from hyperthermia are absent and the incidence of treatment-limiting hot spots should be low. Standard thermometry probes will then be sufficient to monitor treatment quality.

In the future, once the clinical effectiveness of re-irradiation plus hyperthermia for recurrent pediatric sarcoma has been established, the effectiveness of radiotherapy plus hyperthermia for primary tumors may be explored. Radiotherapy may also be necessary in the first-line treatment of childhood sarcomas, particularly when surgery alone (R1/R2 resection) is not sufficient for local tumor control or results in mutilating sequelae. However, radiotherapy is associated with a significant risk of late toxicity, such as atrophy, fibrosis and bone growth abnormalities in the extremities (63). To improve normal tissue sparing and reduce the risk of (late) toxicities, proton beam therapy is emerging, which yields more precisely focused dose distributions, thereby reducing the dose in the low-to-medium dose regions, but not for the OARs in close proximity to the tumor (64). Adding hyperthermia may allow reduction of the delivered dose, thereby significantly reducing the incidence of late side effects.

In conclusion, re-irradiation with $23 \times 2$ Gy plus hyperthermia is a theoretically feasible and possibly effective treatment for recurrent pediatric sarcoma in the pelvic region or the extremities. Hyperthermic radiosensitization is predicted to yield a target-selective additional D95\% of typically $10 \mathrm{~Gy}$, thereby delivering a curative equivalent dose of $54 \mathrm{~Gy}$.

\section{Acknowledgements}

Not applicable.

\section{Funding}

The present study was financially supported by Kika (grant no. 253).

\section{Availability of data and materials}

All data generated or analyzed during the present study are included in this published article.

\section{Authors' contributions}

HPK: Software, investigation, methodology, formal analysis, writing original draft; IWEMvD: Data curation, review and editing; KFC: Investigation, review and editing; NAP: Conceptualization, review and editing; CRN: Conceptualization, funding acquisition, review and editing; JHMM: Conceptualization, funding acquisition, data curation, review and editing. JC: Conceptualization, funding acquisition, review and editing; BVB: Conceptualization, 
data curation, review and editing; AB: Funding acquisition, conceptualization, project administration, review and editing of the manuscript.

\section{Ethics approval and consent to participate}

This retrospective study was not subject to the Medical Research Involving Human Subjects Act and has been conducted in accordance with the ethical standards and according to the principles outlined in the Declaration of Helsinki.

\section{Patient consent for publication}

Consent for the publication of the clinical data was obtained from all patients who were involved in this study.

\section{Competing interests}

J. Crezee, C.R.N. Rasch and A. Bel report a research collaboration with Medlogix, Rome, Italy, outside this study. A. Bel reports grants and non-financial support from Elekta AB, Stockholm, Sweden, outside this work.

\section{References}

1. Kaatsch P: Epidemiology of childhood cancer. Cancer Treat Rev 36: 277-285, 2010.

2. Gatta G, Zigon G, Capocaccia R, Coebergh JW, Desandes E, Kaatsch P, Pastore G, Peris-Bonet R, Stiller CA and Group EW; EUROCARE Working Group: Survival of European children and young adults with cancer diagnosed 1995-2002. Eur J Cancer 45: 992-1005, 2009.

3. Magnani C, Pastore G, Coebergh JW, Viscomi S, Spix C and Steliarova-Foucher E: Trends in survival after childhood cancer in Europe, 1978-1997: Report from the Automated Childhood Cancer Information System project (ACCIS). Eur J Cancer 42: 1981-2005, 2006.

4. Hawkins DS, Spunt SL, Skapek SX and Committee COGSTS; COG Soft Tissue Sarcoma Committee: Children's Oncology Group's 2013 blueprint for research: Soft tissue sarcomas. Pediatr Blood Cancer 60: 1001-1008, 2013.

5. Gorlick R, Janeway K, Lessnick S, Randall RL, Marina N and Committee COGBT; COG Bone Tumor Committee: Children's Oncology Group's 2013 blueprint for research: Bone tumors Pediatr Blood Cancer 60: 1009-1015, 2013.

6. Chisholm JC, Marandet J, Rey A, Scopinaro M, de Toledo JS, Merks JH, O'Meara A, Stevens MC and Oberlin O: Prognostic factors after relapse in nonmetastatic rhabdomyosarcoma: A nomogram to better define patients who can be salvaged with further therapy. J Clin Oncol 29: 1319-1325, 2011.

7. Oeffinger KC, Mertens AC, Sklar CA, Kawashima T, Hudson MM, Meadows AT, Friedman DL, Marina N, Hobbie W, Kadan-Lottick NS, et al; Childhood Cancer Survivor Study: Chronic health conditions in adult survivors of childhood cancer. N Engl J Med 355: 1572-1582, 2006.

8. Geenen MM, Cardous-Ubbink MC, Kremer LC, van den Bos C, van der Pal HJ, Heinen RC, Jaspers MW, Koning CC, Oldenburger F, Langeveld NE, et al: Medical assessment of adverse health outcomes in long-term survivors of childhood cancer. JAMA 297: 2705-2715, 2007.

9. Wust P, Hildebrandt B, Sreenivasa G, Rau B, Gellermann J, Riess H, Felix R and Schlag PM: Hyperthermia in combined treatment of cancer. Lancet Oncol 3: 487-497, 2002.

10. Peeken JC, Vaupel P and Combs SE: Integrating hyperthermia into modern radiation oncology: What Evidence Is Necessary? Front Oncol 7: 132, 2017.

11. Cihoric N, Tsikkinis A, van Rhoon G, Crezee H, Aebersold DM, Bodis S, Beck M, Nadobny J, Budach V, Wust P, et al: Hyperthermia-related clinical trials on cancer treatment within the ClinicalTrials.gov registry. Int J Hyperthermia 31: 609-614, 2015.
12. van der Zee J, González González D, van Rhoon GC, van Dijk JD, van Putten WL and Hart AA; Dutch Deep Hyperthermia Group: Comparison of radiotherapy alone with radiotherapy plus hyperthermia in locally advanced pelvic tumours: A prospective, randomised, multicentre trial. Lancet 355: 1119-1125, 2000.

13. Vernon CC, Hand JW, Field SB, Machin D, Whaley JB, van der Zee J, van Putten WL, van Rhoon GC, van Dijk JD, González González D, et al; International Collaborative Hyperthermia Group: Radiotherapy with or without hyperthermia in the treatment of superficial localized breast cancer: Results from five randomized controlled trials. Int J Radiat Oncol Biol Phys 35: 731-744, 1996.

14. Huilgol NG, Gupta S and Sridhar CR: Hyperthermia with radiation in the treatment of locally advanced head and neck cancer: A report of randomized trial. J Cancer Res Ther 6: 492-496, 2010.

15. Issels RD, Lindner LH, Verweij J, Wessalowski R, Reichardt $P$, Wust P, Ghadjar P, Hohenberger P, Angele M, Salat C, et al; European Organization for the Research and Treatment of Cancer-Soft Tissue and Bone Sarcoma Group and the European Society for Hyperthermic Oncology: Effect of Neoadjuvant Chemotherapy Plus Regional Hyperthermia on Long-term Outcomes Among Patients With Localized High-Risk Soft Tissue Sarcoma: The EORTC 62961-ESHO 95 Randomized Clinical Trial. JAMA Oncol 4: 483-492, 2018.

16. Datta NR, Puric E, Klingbiel D, Gomez S and Bodis S: Hyperthermia and radiation therapy in locoregional recurrent breast cancers: A systematic review and meta-analysis. Int $\mathrm{J}$ Radiat Oncol Biol Phys 94: 1073-1087, 2016.

17. Datta NR, Rogers S, Ordóñez SG, Puric E and Bodis S: Hyperthermia and radiotherapy in the management of head and neck cancers: A systematic review and meta-analysis. Int J Hyperthermia 32: 31-40, 2016.

18. Seifert G, Budach V, Keilholz U, Wust P, Eggert A and Ghadjar P: Regional hyperthermia combined with chemotherapy in paediatric, adolescent and young adult patients: Current and future perspectives. Radiat Oncol 11: 65, 2016.

19. Wessalowski R, Schneider DT, Mils O, Hannen M, Calaminus G, Engelbrecht V, Pape H, Willers R, Engert J, Harms D, et al: An approach for cure: PEI-chemotherapy and regional deep hyperthermia in children and adolescents with unresectable malignant tumors. Klin Padiatr 215: 303-309, 2003.

20. Wessalowski R, Schneider DT, Mils O, Friemann V, Kyrillopoulou O, Schaper J, Matuschek C, Rothe K, Leuschner I, Willers R, et al; MAKEI study group: Regional deep hyperthermia for salvage treatment of children and adolescents with refractory or recurrent non-testicular malignant germ-cell tumours: An open-label, non-randomised, single-institution, phase 2 study. Lancet Oncol 14: 843-852, 2013.

21. de Jong MA, Oldenborg S, Bing Oei S, Griesdoorn V, Kolff MW, Koning CC and van Tienhoven G: Reirradiation and hyperthermia for radiation-associated sarcoma. Cancer 118: 180-187, 2012.

22. Myerson RJ, Roti Roti JL, Moros EG, Straube WL and Xu M: Modelling heat-induced radiosensitization: Clinical implications. Int J Hyperthermia 20: 201-212, 2004.

23. Franken NA, Oei AL, Kok HP, Rodermond HM, Sminia P, Crezee J, Stalpers LJ and Barendsen GW: Cell survival and radiosensitisation: Modulation of the linear and quadratic parameters of the LQ model (Review). Int J Oncol 42: 1501-1515, 2013. (Review).

24. Kok HP, Crezee J, Franken NAP, Stalpers LJA, Barendsen GW and Bel A: Quantifying the combined effect of radiation therapy and hyperthermia in terms of equivalent dose distributions. Int J Radiat Oncol Biol Phys 88: 739-745, 2014.

25. Crezee J, van Leeuwen CM, Oei AL, van Heerden LE, Bel A, Stalpers LJ, Ghadjar P, Franken NA and Kok HP: Biological modelling of the radiation dose escalation effect of regional hyperthermia in cervical cancer. Radiat Oncol 11: 14, 2016.

26. van Leeuwen CM, Crezee J, Oei AL, Franken NA, Stalpers LJ, Bel A and Kok HP: 3D radiobiological evaluation of combined radiotherapy and hyperthermia treatments. Int J Hyperthermia 33: 160-169, 2017.

27. International Commission on Radiation Units and Measurements: ICRU Report 50. Prescribing, recording, and reporting photon beam therapy. ICRU, Bethesda, MD, 1993.

28. van Dijk JDP, Schneider C, van Os R, Blank LE and Gonzalez DG: Results of deep body hyperthermia with large waveguide radiators. Adv Exp Med Biol 267: 315-319, 1990. 
29. van Stam G, Kok HP, Hulshof MCCM, Kolff MW, van Tienhoven G, Sijbrands J, Bakker A, Zum Vörde Sive Vörding PJ, Oldenborg S, de Greef M, et al: A flexible $70 \mathrm{MHz}$ phase-controlled double waveguide system for hyperthermia treatment of superficial tumours with deep infiltration. Int J Hyperthermia 33: 796-809, 2017.

30. Kok HP, Kotte ANTJ and Crezee J: Planning, optimisation and evaluation of hyperthermia treatments. Int J Hyperthermia 33: 593-607, 2017.

31. Taflove A and Hagness SC: Computational Electrodynamics. 2nd edition. Artech House, Boston, London, 2000.

32. Das SK, Clegg ST and Samulski TV: Computational techniques for fast hyperthermia temperature optimization. Med Phys 26 319-328, 1999.

33. Stoll AM and Greene LC: Relationship between pain and tissue damage due to thermal radiation. J Appl Physiol 14: 373-382, 1959.

34. van Leeuwen CM, Oei AL, Ten Cate R, Franken NA, Bel A, Stalpers LJ, Crezee J and Kok HP: Measurement and analysis of the impact of time-interval, temperature and radiation dose on tumour cell survival and its application in thermoradiotherapy plan evaluation. Int J Hyperthermia 34: 30-38, 2018.

35. Bruggmoser G, Bauchowitz S, Canters R, Crezee H, Ehmann M, Gellermann J, Lamprecht U, Lomax N, Messmer MB, Ott O, et al; Atzelsberg Research Group; European Society for Hyperthermic Oncology: Guideline for the clinical application, documentation and analysis of clinical studies for regional deep hyperthermia: Quality management in regional deep hyperthermia. Strahlenther Onkol 188 (Suppl 2): 198-211, 2012.

36. International Commission on Radiation Units and Measurements: ICRU report 83. Prescribing, Recording, and Reporting Intensity-Modulated Photon-Beam Therapy (IMRT). ICRU, Bethesda, MD, 2010.

37. van Leeuwen CM, Oei AL, Crezee J, Bel A, Franken NA, Stalpers LJ and Kok HP: The alfa and beta of tumours: A review of parameters of the linear-quadratic model, derived from clinical radiotherapy studies. Radiat Oncol 13: 96, 2018.

38. van Dijk IW, van Os RM, van de Kamer JB, Franken NA, van der Pal HJ, Koning CC, Caron HN, Ronckers CM and Kremer LC: The use of equivalent radiation dose in the evaluation of late effects after childhood cancer treatment. J Cancer Surviv 8: 638-646, 2014.

39. Brahme A: Individualizing cancer treatment: Biological optimization models in treatment planning and delivery. Int J Radiat Oncol Biol Phys 49: 327-337, 2001.

40. Kaidar-Person O, Oldenborg S and Poortmans P: Re-irradiation and Hyperthermia in Breast Cancer. Clin Oncol (R Coll Radiol) 30: 73-84, 2018.

41. Overgaard J: Simultaneous and sequential hyperthermia and radiation treatment of an experimental tumor and its surrounding normal tissue in vivo. Int J Radiat Oncol Biol Phys 6: 1507-1517, 1980.

42. van Leeuwen CM, Crezee J, Oei AL, Franken NA, Stalpers LJ, Bel A and Kok HP: The effect of time interval between radiotherapy and hyperthermia on planned equivalent radiation dose. Int J Hyperthermia 34: 901-909, 2018.

43. ESHO Taskgroup Committee: 'Treatment Planning and Modelling in Hyperthermia, a Task Group Report of the European Society for Hyperthermic Oncology. Tor Vergata, Rome, 1992.

44. de Greef M, Kok HP, Correia D, Borsboom PP, Bel A and Crezee J: Uncertainty in hyperthermia treatment planning: The need for robust system design. Phys Med Biol 56: 3233-3250, 2011.

45. Dewhirst MW, Vujaskovic Z, Jones E and Thrall D: Re-setting the biologic rationale for thermal therapy. Int J Hyperthermia 21: 779-790, 2005

46. Sreenivasa G, Gellermann J, Rau B, Nadobny J, Schlag P, Deuflhard P,Felix R and Wust P: Clinical use of the hyperthermia treatment planning system HyperPlan to predict effectiveness and toxicity. Int J Radiat Oncol Biol Phys 55: 407-419, 2003.
47. Willnow U, Lindner H, Brock D, Wild L, Diestelhorst C, Greiner $\mathrm{C}$ and Eichstädt $\mathrm{H}$ : Treatment of otherwise incurable tumor diseases in childhood using whole-body hyperthermia and chemotherapy. Dtsch Med Wochenschr 114: 208-213, 1989 (In German).

48. Lindner $\mathrm{H}$ and Tillig B: Treatment of recurrent neuroblastoma in childhood with whole body thermochemotherapy. Padiatr Grenzgeb 31: 187-194, 1993 (In German).

49. Ismail-Zade RS, Zhavrid EA and Potapnev MP: Whole body hyperthermia in adjuvant therapy of children with renal cell carcinoma. Pediatr Blood Cancer 44: 679-681, 2005.

50. Geijsen ED, de Reijke TM, Koning CCE, Zum Vörde Sive Vörding PJ, de la Rosette JJ, Rasch CRN, van Os RM and Crezee J: Combining Mitomycin C and Regional $70 \mathrm{MHz}$ Hyperthermia in Patients with Nonmuscle Invasive Bladder Cancer: A Pilot Study. J Urol 194: 1202-1208, 2015

51. Hulshof MC, Van Haaren PM, Van Lanschot JJ, Richel DJ, Fockens P, Oldenborg S, Geijsen ED, Van Berge Henegouwen MI and Crezee J: Preoperative chemoradiation combined with regional hyperthermia for patients with resectable esophageal cancer. Int J Hyperthermia 25: 79-85, 2009.

52. Turner PF, Tumeh A and Schaefermeyer T: BSD-2000 approach for deep local and regional hyperthermia: Physics and technology. Strahlenther Onkol 165: 738-741, 1989.

53. Horsman MR and Overgaard J: Hyperthermia: A potent enhancer of radiotherapy. Clin Oncol (R Coll Radiol) 19: 418-426, 2007.

54. Sapareto SA and Dewey WC: Thermal dose determination in cancer therapy. Int J Radiat Oncol Biol Phys 10: 787-800, 1984.

55. Dewey WC, Hopwood LE, Sapareto SA and Gerweck LE: Cellular responses to combinations of hyperthermia and radiation. Radiology 123: 463-474, 1977.

56. Sapareto SA, Hopwood LE and Dewey WC: Combined effects of $\mathrm{X}$ irradiation and hyperthermia on $\mathrm{CHO}$ cells for various temperatures and orders of application. Radiat Res 73: 221-233, 1978.

57. Crezee H, van Leeuwen CM, Oei AL, Stalpers LJ, Bel A, Franken NA and Kok HP: Thermoradiotherapy planning: Integration in routine clinical practice. Int J Hyperthermia 32: 41-49, 2016

58. Wijsman R, Kaanders JH, Oyen WJ and Bussink J: Hypoxia and tumor metabolism in radiation oncology: Targets visualized by positron emission tomography. Q J Nucl Med Mol Imaging 57: 244-256, 2013.

59. Niendorf T, Pohlmann A, Arakelyan K, Flemming B, Cantow K, Hentschel J, Grosenick D, Ladwig M, Reimann H, Klix S, et al: How bold is blood oxygenation level-dependent (BOLD) magnetic resonance imaging of the kidney? Opportunities, challenges and future directions. Acta Physiol (Oxf) 213: 19-38, 2015.

60. Kok HP: Korshuize - van Straten L, Bakker A, De Kroon-Oldenhof R, Geijsen ED, Stalpers LJA, and Crezee J, On-line adaptive hyperthermia treatment planning during locoregional heating to suppress treatment limiting hot spots. Int J Radiat Oncol Biol Phys 99: 1039-1047, 2017.

61. Balzer S, Schneider DT, Bernbeck MB, Jäger M, Mils O, Schaper J, Willers R, Krauspe R, Göbel U and Wessalowski R: Avascular osteonecrosis after hyperthermia in children and adolescents with pelvic malignancies: A retrospective analysis of potential risk factors. Int J Hyperthermia 22: 451-461, 2006.

62. Jäger M, Balzer S, Wessalowski R, Schaper J, Göbel U, Li X and Krauspe R: Hyperthermia associated osteonecrosis in young patients with pelvic malignancies. Anticancer Agents Med Chem 8: 571-575, 2008.

63. Paulino AC: Late effects of radiotherapy for pediatric extremity sarcomas. Int J Radiat Oncol Biol Phys 60: 265-274, 2004.

64. DeLaney TF and Haas RL: Innovative radiotherapy of sarcoma: Proton beam radiation. Eur J Cancer 62: 112-123, 2016. 